Article

\title{
An Experimental Study on the Water Hammer with Cavity Collapse under Multiple Interruptions
}

\author{
Li Zhao ${ }^{1, *}$, Yusi Yang ${ }^{2}$, Tong Wang ${ }^{2}$, Wensheng Han ${ }^{2}$, Rongchu $\mathrm{Wu}^{3}$, Pengli Wang ${ }^{4}$, \\ Qiaoning Wang ${ }^{1}$ and Liang Zhou ${ }^{1}$ \\ 1 School of Energy and Architecture, Xi'an Aeronautical University, Xi'an 710077, China; \\ wqnlily@163.com (Q.W.); zhouliang@xaau.edu.cn (L.Z.) \\ 2 Key Laboratory of Water Supply and Drainage, School of Architecture and Engineering, \\ Ministry of Housing and Urban-Rural Development, Chang'an University, Xi'an 710054, China; \\ yangys@chd.edu.cn (Y.Y.); wangt@chd.edu.cn (T.W.); wenshhan@chd.edu.cn (W.H.) \\ 3 Guangzhou Metro Design \& Research Institute Co., Ltd. Guangzhou 510000, China; wurongchu@163.com \\ 4 China Airport Construction Group Co., Ltd. Xi'an 710054, China; pengliwang000@163.com \\ * Correspondence: zhaoli200817@163.com; Tel.: +86-15339097446
}

Received: 29 June 2020; Accepted: 11 September 2020; Published: 14 September 2020

check for updates

\begin{abstract}
Pressurized pipeline system damage is primarily caused by the highly destructive water hammer force. Currently, research on water hammer-caused collapse is mostly based on single-point collapse cases, but water hammer research, which involves multipoint collapse, is insufficient. Here, we establish an experimental platform to realize water hammers with multipoint collapse. With different schemes, i.e., various initial flow rates and valve closing speeds, we observed the hydraulic transient process with a high-speed camera, analyzed its characteristics and explained experimental phenomena with theoretical knowledge. Using experimental data analysis, we summarized the influencing factors and laws of the cavity length and water hammer pressure. Flow and pressure data for the different schemes were recorded to provide basic simulation data. Water column separation experimental phenomena were observed: completely atomized, completely cavitated and partially cavitated, and both cavitated and atomized. At the pump outlet, three hydraulic transition states occurred simultaneously in the horizontal pipe section: completely atomized, completely cavitated, and both cavitated and atomized. Two hydraulic transition states occurred in the knee region: completely and partially cavitated, and without atomization. The experimental results reveal that the initial flow rate and valve closing speed greatly affect the water hammer pressure rise and cavity length. The higher the initial flow rate and valve closing speed are, the larger the water hammer pressure rise and cavity length are.
\end{abstract}

Keywords: water hammer; transient flow; water column separation; cavity collapse; experimental model

\section{Introduction}

With the rapid development of long-distance water conveyance projects, their safety guarantee is becoming increasingly important. Because of the complexity of the water hammer phenomenon, researchers do not fully understand it, especially when water hammers with multipoint collapse occur in a pipeline system, which highly complicates the situation [1,2]. The water hammer phenomenon with multipoint collapse is the main cause of pipe burst accidents [3]. Due to the complexity of the water hammer with multipoint collapse, relevant formation mechanisms and influencing factors remain unclear [4]. In certain conditions, such as when a pipeline valve is quickly opened or closed or when a pump is accidentally activated or stopped, water hammer pressure fluctuations may occur $[5,6]$. When the pressure in the pipe drops to the vapor pressure level of the liquid, it will 
vaporize, and a vapor-filled cavity is created, which can be large enough to occupy the whole pipe cross-section and cause fluid discontinuity. Under the action of water hammer waves, these vapor cavities gradually decrease in size and eventually collapse, which causes a sudden pressure peak [7]. Usually, these cavities will stretch and shrink repeatedly until they collapse. The pressure peak caused by cavity collapse will often cause pipeline system failure and even cause a pipe to burst [8]. When the pump is stopped in the case of an accident, the valve closing speed at the pump outlet and the initial flow rate in the pipeline are important factors that influence the water hammer pressure. Most previous studies have been based on the case of a water hammer at a single point [9], but water hammers at multiple points need to be further studied theoretically and experimentally. An experimental platform was established to study the characteristics of the water hammer with multipoint collapse. The flow pattern due to hydraulic transition was observed, and the experimental phenomena were explained based on theoretical knowledge. The factors that impact the cavity length and water hammer pressure were analyzed with the experimental data. Experimentation is very important to better understand the mechanism of the water hammer with multipoint collapse and to establish a better calculation model [10].

\section{Materials and Methods}

\subsection{Experimental Device}

The experimental system was composed of an upper water tank, lower water tank, water pumps, pipeline, various valves, pressure gauges, electromagnetic flowmeter, pressure sensors, and a water pump outlet quick shut-off valve. At the pump outlet, a self-developed quick-closing valve that can realize a valve closing time in the range from $0.2-5 \mathrm{~s}$, at a certain precision attained via calibration, was installed. The electromagnetic flowmeter and pressure sensors were arranged at specific positions along the pipeline, and the pressure and flow rate at the set points were accurately recorded with a LabVIEW numerical control system.

As shown in Figure 1, the water delivery system pumps water from the lower tank (simulated water source) to the upper tank. The relative elevation of the lower water tank is $2.6 \mathrm{~m}$, and that of the upper water tank is $15.6 \mathrm{~m}$. There are four observation sections, which consist of plexiglass pipe, and the total length reaches $20 \mathrm{~m}$, which can realize the simultaneous occurrence and observation of the water hammer phenomenon with multipoint collapse. There are 10 pressure detection points distributed along the water delivery pipeline, where the pipeline pressure is monitored in real time, and the data are transferred to a computer for subsequent analysis. The pipe system is composed of DN100 galvanized steel segments and DN100 plexiglass pipe segments. The length of each pipe segment is one meter. The pipe segments are connected by clamps [4]. The two pumps can supply water in series or in parallel, which satisfies the requirements of different flow rates. The main materials and equipment and physical drawings of some of the equipment are described in the supplementary information (Table S1, Figures S1-S6). 


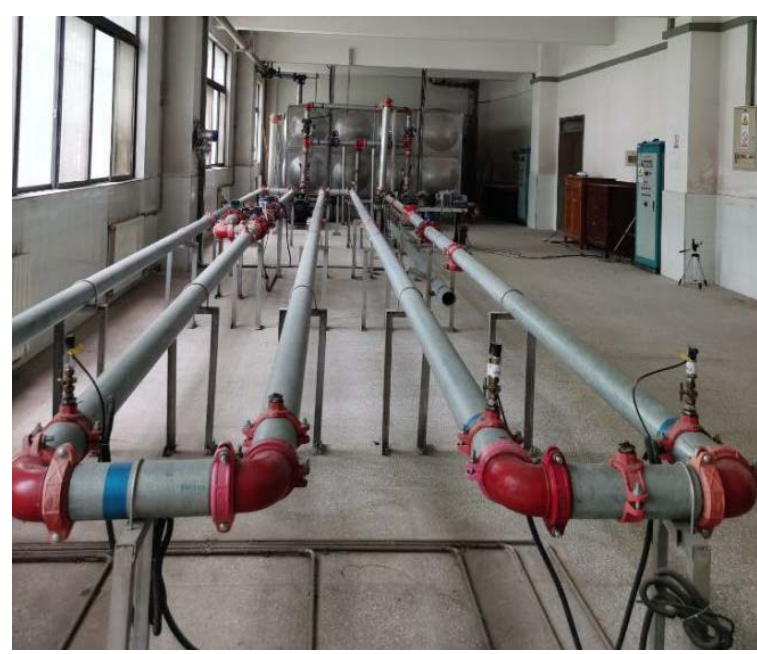

(a)

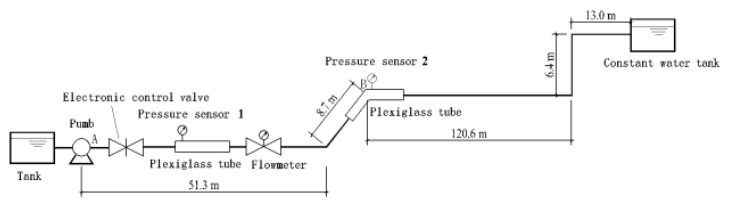

(b)

Figure 1. Photo (a) and schematic (b) of the experimental device.

\subsection{Preparation of the Experiment and Establishment of the Experimental Plan}

The preparation work before the experiment included the calibration of the instruments and flowmeters and the measurement of the wave velocity and resistance coefficient along the pipeline [11,12]. The experimental scheme was developed based on the pre-experiments. To better observe and assess the phenomenon of water column interruption and detect the pressure rise due to the water hammer with multipoint collapse in various states, different initial flow rates in the pipeline system and closing velocities of the electric control valve at the pump outlet were tested with various experimental schemes. The hydraulic transition phenomenon in the pipeline system was observed, and flow and pressure data were recorded. An experimental pipeline system diagram is shown in Figure 2. The total length of the pipeline system is $200 \mathrm{~m}$. Pipe knee point B (No. 2 pressure point) represents the boundary, which divides the pipeline into two sections, namely, the upstream pipeline section and downstream pipeline section. The length of the pipeline is $60 \mathrm{~m}$ from Point 1 to Point 2 and $140 \mathrm{~m}$ from Point 2 to the upper water tank at the end of the pipeline (Figure 1b). The unified valve shut-off time was set to $1 \mathrm{~s}$. By manually adjusting the valve opening at the pump outlet, the flow rate can be controlled to 58 , $65,70.75,74$ and $77.7 \mathrm{~m}^{3} / \mathrm{h}$; the initial flow rate was set to $70 \mathrm{~m}^{3} / \mathrm{h}$; and the valve closing times were 0.6 , $0.7,0.8,0.9,0.95$ and $1 \mathrm{~s}$, respectively.

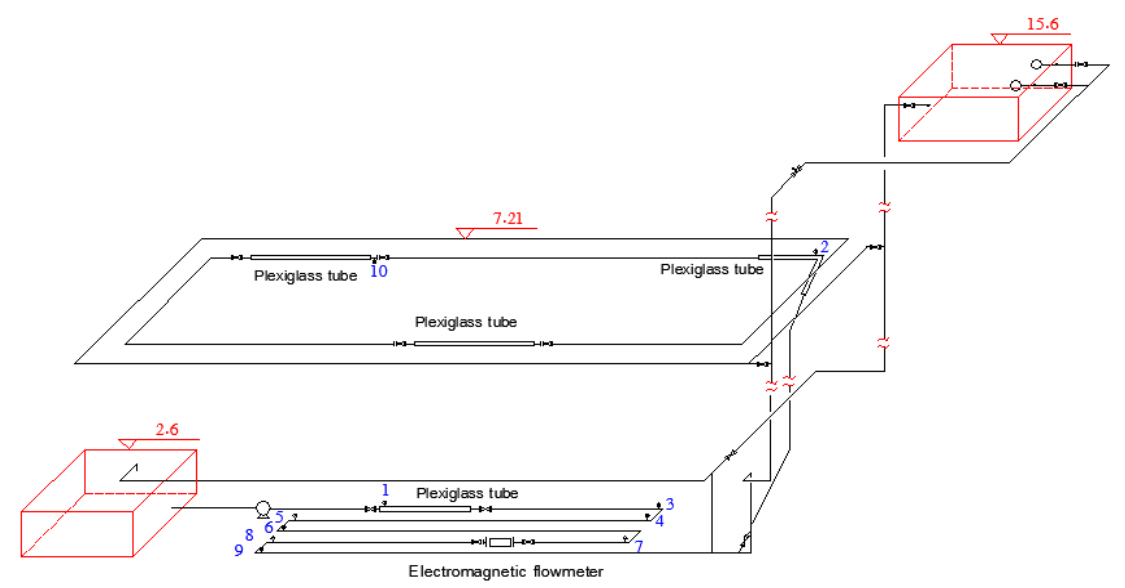

Figure 2. Schematic of the experimental pipeline system. Note: The blue numbers indicate the position of the pressure sensor. 


\subsubsection{Calibration of the Instruments and Meters}

An Omega pressure sensor was employed in the experiment. The range is -1 bar-15 bar, and the position of the sensor is shown in Figure 2. According to the measurement principle and instructions, the physical quantities measured by the electromagnetic flowmeter and pressure sensor are linearly related to the output signal; thus, it is not difficult to calibrate in a steady state. The Krohne electromagnetic flowmeter is calibrated before it leaves the factory; therefore, we needed only to check that the electromagnetic flowmeter head pressure was consistent with the stored value in Labview to ensure the correctness of transmission. The pressure sensor can be calibrated using a pressure gauge, and a check also needs to be performed to ensure that the pressure sensor is consistent with the stored value in Labview. The speed control box of the electric control valve controls the valve closing speed by adjusting the speed of the motor; the closing time is determined by the valve that approaches the iron plate of the switch induction terminal; and the closing time data is stored in Labview.

\subsubsection{Determination of the Resistance Coefficient Along the Pipeline}

Taking Sections 1-1 and 2-2 in horizontal pipeline, the Bernoulli equations for Sections 1-1 and 2-2 are given by selecting the pipe section between two pressure sensors.

$$
Z_{1}+\frac{P_{1}}{\rho g}+\frac{\alpha_{1} v_{1}^{2}}{2 g}=Z_{2}+\frac{P_{2}}{\rho g}+\frac{\alpha_{2} v_{2}^{2}}{2 g}+h_{f}+h_{j}
$$

where the Darcy-Weisbach formula is: $h_{f}=\lambda \frac{l}{d} \frac{v^{2}}{2 g} ; l$ : pipe length $(\mathrm{m}) ; h_{f}$ : head loss (m); $d$ : hydraulic diameter $(\mathrm{m}) ; \lambda$ : drag coefficient $(\mathrm{m}) ; g$ : gravitational acceleration $\left(\mathrm{m} / \mathrm{s}^{2}\right)$; and $v$ : flow velocity $(\mathrm{m} / \mathrm{s})$. As the elevation and flow velocity of Sections 1-1 and 2-2 are equal, the drag coefficient formula is:

$$
\lambda=\frac{\left(P_{1}-P_{2}\right) 2 g d}{v^{2} l}
$$

The measured values of $\mathrm{P}_{1}, \mathrm{P}_{2}$ and $v$ are input into formula (2), and the drag coefficient for each flow rate is calculated. The drag coefficients are then added to an average value, and the absolute value of the relative error is obtained by dividing the difference between the drag coefficient and the average value at each velocity. The average resistance coefficient of a PMMA pipe is 0.01905, and the average resistance coefficient of the steel tube is 0.0318 .

For the method that is employed to measure the local resistance coefficient of the elbow, there are two 90-degree elbows between two sensors. The value of the upstream pressure sensor is d; the value of the downstream pressure sensor is e; the length of the horizontal pipe is $\mathrm{L}$; and the pressure difference of the pressure sensor minus the head loss of the horizontal steel pipe is the local loss between the two 90-degree elbows. The formula for calculating the partial loss is expressed as follows:

$$
h_{j}=\frac{\zeta v^{2}}{2 g}
$$

The formula of the local resistance coefficient is arranged as:

$$
\zeta=\frac{2 g\left(10.33 \times d-10.33 \times e-\lambda \frac{L v^{2}}{2 d g}\right)}{v^{2}}
$$

The measured values are input into Equation (4), the corresponding local resistance coefficients for each flow rate are calculated, and then these local resistance coefficients are added to an average value. The absolute value of the relative error is obtained by dividing the difference between the local resistance coefficient and the average value at each flow rate.

The average resistance coefficient of a 90-degree elbow is 0.4921 , and that of a 45-degree elbow is 0.2686 . 


\subsubsection{Determination of the Water Hammer Wave Velocity}

In the vicinity of the pump outlet, the valve was quickly closed; a water hammer fluctuation was implemented; and the time interval of the pressure change between the two pressure measuring points on the tube, i.e., the propagation time of the water hammer wave, was calculated. The velocity of the water hammer wave in the tube could be obtained by dividing the path length between the two pressure measuring points by the propagation time of the water hammer wave between those two points. The measured wave velocity for the plexiglass tube and the steel tube was $500.7 \mathrm{~m} / \mathrm{s}$ and $1221.7 \mathrm{~m} / \mathrm{s}$, respectively. The wave velocity measurement data are provided in the supplementary information (Tables S2 and S3).

\subsubsection{Determination of the Resistance Coefficient of the Valves}

In the experiment, a homemade fast-closing electric valve is applied to close the valve to create a hydraulic transition state. The resistance coefficient of the valve is the main parameter in the simulation calculation; hence, the head loss of the valve with different opening degrees was measured to give the valve opening and the discrete value of the resistance coefficient for the simulation calculation. The method is similar to the measurement of the local resistance coefficient, that is, the difference in the pressure between the two pressure sensors of the valve was deducted to obtain the measured local loss of the valve.

In the process of steady-state operation, the measured value of the pressure sensor at the pressure measuring point is changed by adjusting the valve opening. The pressure and flow value after the valve opening is adjusted and recorded, and the resistance coefficient of the valve is calculated by using the local loss formula (3). To avoid the operating error of the valve, the average value of the resistance coefficient of the valve closing and opening process was taken as the resistance coefficient of the corresponding opening degree. The relationship curve between the resistance coefficient and the opening degree is given in the supplementary information (Tables S4-S6, Figure S7).

Note: In this experiment, the valve shut-off is extremely fast, the main purpose is to produce water hammer with cavity collapse under multiple interruptions. Usually, the reflected wave has little effect on the resistance of the valve, thus the transient flow-rate curve of a valve [13] and the unsteady friction are not the main contents of the experiment; the purpose of measuring the resistance characteristic curve of the valve in this experiment is only to provide the basic data for the simulation calculation in the special case of this experiment (when the valve closes extremely fast).

\subsection{Experimental Operation}

After the pump is started and operates normally, the manual gate valve that is located near the pump outlet is adjusted to obtain a stable initial flow rate throughout the tubeline system [14]. Before each valve shut-off, steady operation of more than $30 \mathrm{~min}$ was ensured to guarantee the removal of free gas that remains within the tubeline system. After the initial state has been determined, the valve speed was adjusted according to the requirements, and the steering switch was pulled such that the electric control valve was in an open-to-close state. When the system was ready, the remote control switch of the electric control valve was activated to realize a water hammer with multipoint collapse. In addition, the water column separation phenomena were observed, and the separation length, pressure, and flow rate were measured. During the experiment, data and images were recorded in real time and stored on a computer $[9,14]$. After the first experiment, the control box was switched off to prevent it from burning. Moreover, the motor could be reversed to open the electric control valve, which was readied for the next experiment [14].

\section{Analysis of the Experimental Phenomena}

The liquid column separation phenomenon caused by the pressure transient in the pressurized tube flow is an important problem in hydraulic transient analysis. As it is the foundation of the physical 
model, this phenomenon determines whether the mathematical simulation is correct. By changing the initial flow rate and valve closing speed, we evaluated the experimental phenomena and measured data and summarized the water hammer influencing factors and control mechanism. When the pump outlet quick closing control valve closes, the water in the tubeline continued to flow forward depending on the inertial effect. However, as the valve closes and there was no additional water behind it, the pressure at the valve outlet was reduced and the water body vaporized. When the pressure-drop wave vaporized part of the water, the water column still had some residual velocity, the water column still continued to advance at high speed, and the water body had no time to vaporize; thus, the partial cavity will appear. The following section provides a comprehensive description of the various water column separation phenomena observed in the experiments.

\subsection{Completely Atomised Vaporisation State}

This condition is only observed in the first section after the electric control valve outlet in the horizontal observation tube section (Point 1). When the valve closing time ranges from 1.0-1.5 s, complete atomization occurs, and the higher the initial flow velocity is, the longer the atomization zone will be. With the change in the pressure wave, the cavity collapses $2-4$ times and completely disappears (Figure 3).

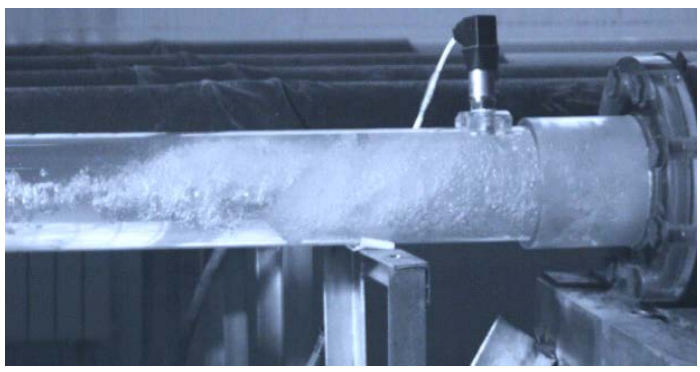

Figure 3. Completely atomized vaporization state.

\subsection{State with Both Flow Interruption Cavity and Atomized Vaporisation Zones}

This state is only observed in the horizontal tube section downstream of the electric control valve. This state only occurs at the electric control valve after the horizontal tube section (Point 1 ), and a fast valve shut-off situation (the shut-off time ranges from 0.8-1.0 s) is observed. After the water is partially vaporized by the depressurization wave, the water column still exhibits a residual velocity, and the water column continues to advance at a high speed. The water does not have time to completely vaporize, and at this time, a partial cavity is observed. Between the cavity and the water column, a fog-like vaporization zone is observed. The surface of the cavity is sloped and concentrated along the top of the tube, as shown in Figure 4. The water surface below the cavity extends from the atomized vaporization zone to the electric control valve. However, a complete flow interruption state is not formed. The valve closing speed in this state is higher than that in the completely atomized vaporization state.

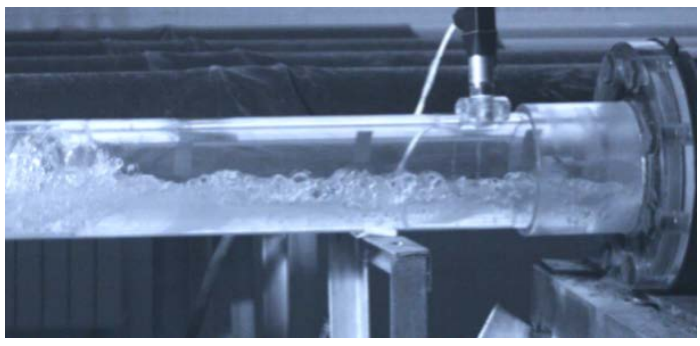

Figure 4. State with both flow interruption cavity and atomized vaporization zones. 


\subsection{Complete Flow Interruption State}

This hydraulic transition state occurs mostly at Point 2 of the knee in the middle of the tubeline, as shown in Figure 5b. The cavity causes a complete interruption and cuts the original continuous water column into two sections, while a distinct slope is observed in the horizontal tube downstream of the separation point. The liquid level in the inclined tube section at the upstream end of the separation point is almost horizontal. The first impingement point of the two separated water columns generally occurs on the upstream side of the separation point but gradually moves back to the downstream end of the separation point over time. The number of impingement points usually ranges from 2-4. A complete flow interruption state may also be observed in the horizontal segment. When the initial flow rate is higher than $70 \mathrm{~m}^{3} / \mathrm{h}$ and the valve closing time is shorter than $1 \mathrm{~s}$, the complete flow interruption state will occur in the horizontal section (Point 1) at the outlet of the electric control valve, as shown in Figure 5a. The slope of the liquid surface below the interrupted flow cavity in the horizontal section is larger than that in the knee section of the tubeline, and a short atomized vaporization zone is observed downstream of the cavity.

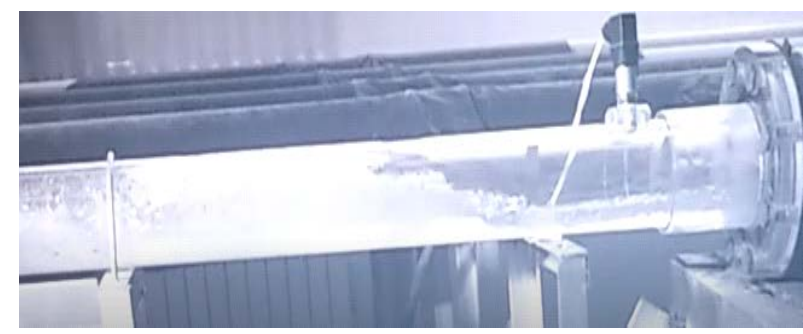

(a)

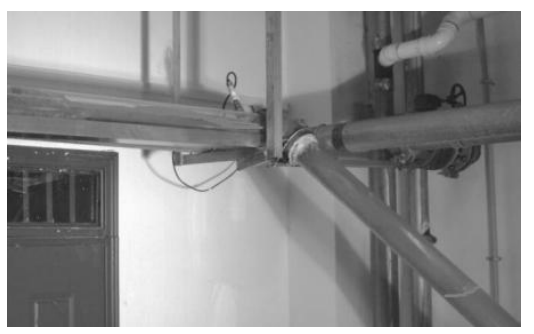

(b)

Figure 5. Complete flow interruption state: (a) the complete flow interruption state at Point 1; and (b) the complete flow interruption state at Point 2.

\subsection{Incomplete Flow Interruption State}

This hydraulic transition state mostly occurs at Point 2 of the knee in the middle of the tubeline, as shown in Figure 6. When the initial flow rate is lower than $2.5 \mathrm{~m} / \mathrm{s}$ and the valve closing time is longer than $1 \mathrm{~s}$, an incomplete flow interruption state is observed in the knee section of the tubeline (Point 2). However, in contrast to the horizontal section (Point 1), there is no distinct atomized vaporization zone in the knee section of the tubeline. Only a cavity at the top of the tube occurs or discontinuous bubbles of different sizes are observed. The bubbles collapse and disappear rapidly, which causes an increase in pressure.

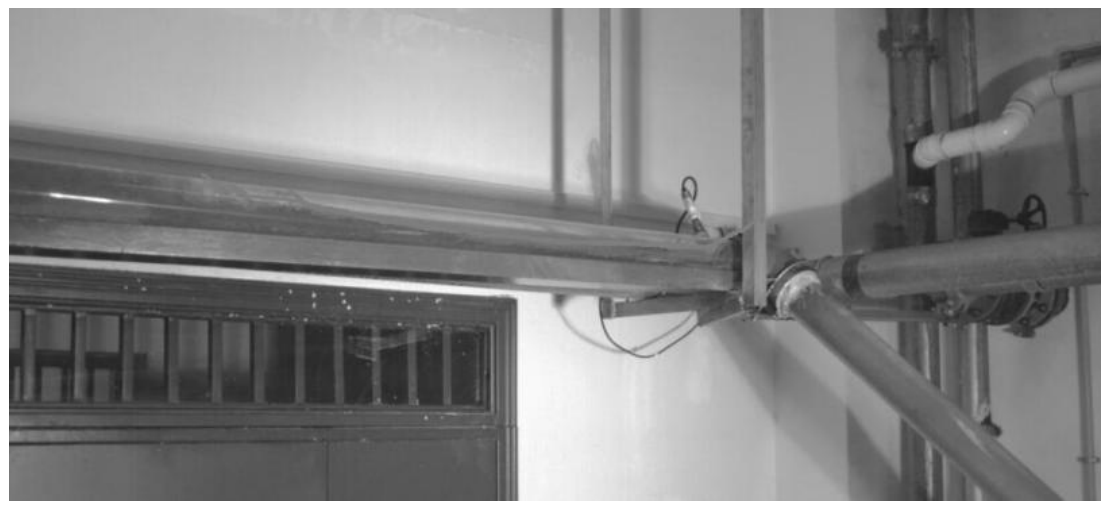

Figure 6. Incomplete interruption at Point 2 at the knee.

The four types of water column separation (interruption) conditions that have been described are affected by the initial flow rate, valve closing speed, and location of the interruption zone. The transient 
states observed in the horizontal tubeline (Point 1) include the completely atomized vaporization state, the state with both flow interruption cavity and atomized vaporization zones, and the complete and incomplete flow interruption states. The transient states observed at the knee of the tubeline (Point 2) include the complete and incomplete flow interruption states (or only a few bubbles occur). Two interesting phenomena are observed in the experiments. First, in the cavity zone in observation tube section B at the knee of the second level, the atomized vaporization state is never observed in any experimental condition. Second, the atomized vaporization zone is always observed at Point 1 in the horizontal observation section on the first level at the valve outlet.

\section{Discussion}

\subsection{Causes of the Different Flow Interruption States}

When the initial flow rate and valve closing speed are gradually increased, different hydraulic transition flow states may occur in the tubeline system. The basic reason for a pressure transient in pressurized tube flow is a sudden change in the flow velocity in the tube. According to the Joukowsky equation $[14,15]$, the change in flow velocity will inevitably cause a change in pressure. In the experiments, due to the fast closing of the valve, the flow rate in the tube rapidly decreases, which causes a rapid drop in pressure. When the pressure is reduced to the liquid vaporization pressure, the flow state is determined by the remaining flow. If the residual flow is sufficient, complete flow interruption can occur. If the residual flow is low or even approaches 0 , incomplete flow interruption or atomized vaporization might occur. If the vaporization pressure is maintained for a short time, it is difficult to observe the atomized vaporization phenomenon. The residual flow depends on both the initial flow rate and the pressure increase wave reflected from the end of the tubeline, which explains why the flow interruption state is controlled by the initial flow rate, valve closing speed, and interruption position.

\subsection{Interpretation of the Cavity in the Incomplete Flow Interruption State Observed at the Top of the Tube}

When incomplete flow interruption occurred in the horizontal tubeline (Point 1) or at the knee of the tubeline (Point 2), the cavity in the incomplete flow interruption state was always observed at the top of the tube. Theoretically, the phenomenon might be interpreted as follows: gas (air or water vapor) is lighter than water, and gas thus floats upwards. However, the experimental results indicated that in the process of an instantaneous interruption, even if bubbles were present, they could not immediately rise to the top. Therefore, this phenomenon could only be interpreted from the perspective of the gravity distribution of water. During normal operation, the flow of water in the tubeline remained stable, and the flow rate was evenly distributed across the tubeline cross-section. When the valve was closed, the pressure-relief wave reduced the pressure in the tube below the vaporization pressure of water. At this time, the water body exhibited a sufficient residual flow rate [14], which forms the liquid column separation phenomenon. In the horizontal tubeline section (Point 1) connected to the valve outlet on the first level, when the valve was closed, the liquid flow rate was nil on one side of the valve, whereas a certain residual flow rate occurred on the other side due to the inertia of the water body. However, the distribution of this residual flow rate across the tubeline cross-section was not uniform. With the change in the height of the water level section, the flow rate constantly varied. Due to the uneven distribution because of gravity, the flow rate of the water body closer to the top of the tube was higher. In the hydraulic transient process, once a cavity was formed, the gas-liquid interface exhibited a sloped surface and the phenomenon, whereby the cavity length closer to the top of the tube was longer.

The flow velocity at the top of the tube is always higher than that at the bottom of the tube, which causes the flow interruption cavity to form a sloped surface. When the residual flow velocity is low enough due to the reflected waves, the flow interruption cavity is only formed at the top of the tube. Regarding the cavity in a large-diameter tube, with an increase in the tube diameter, it becomes 
more difficult to form a complete flow interruption cavity. For an incomplete flow interruption cavity, in the hydraulic transient process, a preliminary estimate of the force during the cavity collapse process is shown in Figure 7. The cavity is attached to the top of the tube via a thin layer. In the cavity collapse process, the following situation is more common: the upward impact of the water flow below the cavity causes it to quickly collide with the contact surface at the tube top, which substantially increases the pressure. The pressure boost is more destructive than the water column collapse phenomenon. Therefore, for the same cavity volume, tubes with large diameters experience a high burst probability. This qualitative analysis requires further experimental results, but it is difficult to establish the required experimental platform.

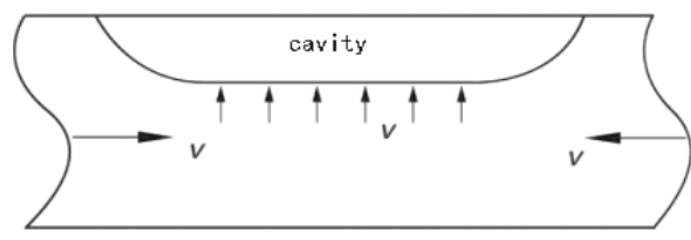

Figure 7. Force analysis of the cavity collapse process in a large-diameter tube.

\subsection{Analysis of the Absence of a Fog Vaporisation Zone at the Knee (Point 2) of the Tubeline}

When the pressure drops, the mixture of water and vapor produced by water vaporization under low pressure and the expansion of small bubbles constitute the essence of the atomized vaporization zone. The vaporization pressure of water at room temperature is close to the critical pressure of small bubbles. It can be considered that when the pressure reaches the vaporization pressure, the atomized vaporization zone will only occur at a low residual flow velocity. In the horizontal tube section (Point 1) at the outlet of the quick-closing valve, the gravity gradient is low, the flow velocity changes very gradually, and the vaporization pressure can be maintained for a certain period. In contrast, the situation at Point 2 is different, which depends on both inertia and gravity. The gravity gradient at the knee of the tubeline (point 2) is high, and the corresponding flow change is rapid. The gravity gradient at the knee of the tubeline (point 2) is high, the flow velocity changes quickly, and the time for the pressure-drop wave to affect the flow at point 2 is very short. Due to the gravity effect of the water in the inclined tube segment upstream of point 2, the velocity rapidly decreases. However, the horizontal tube segment downstream of point 2 is only slightly affected by gravity, and the inertia of the water flow has a major role. A flow velocity difference between the two water columns is quickly established, which causes the water flow to break; a distinct flow interruption cavity is observed.

\subsection{Analysis of the Experimental Data}

According to the experimental protocol, via experiments, the effects of the initial flow rate and valve closing speed on the cavity length and pressure increase were examined. The horizontal observation tube section (No. 1 pressure sensor) at the pump outlet on the first floor and observation tube section (No. 2 pressure sensor) at the knee of the second floor were taken as the research objects. Data from the No. 1 and No. 2 pressure sensors were recorded with different flow rates and valve closing schemes. According to the measured pressure data, the maximum value of the pressure in the measured working condition was obtained. Simultaneously, the maximum cavity length was recorded by a high-speed camera in the corresponding working conditions. The measured pressure data at a flow rate of $77 \mathrm{~m}^{3} / \mathrm{h}$ and a valve closing time $1 \mathrm{~s}$ are listed in Figures 8 and 9. In addition, In addition, 5 groups of parallel tests were carried out under each condition, and the average values were recorded. 


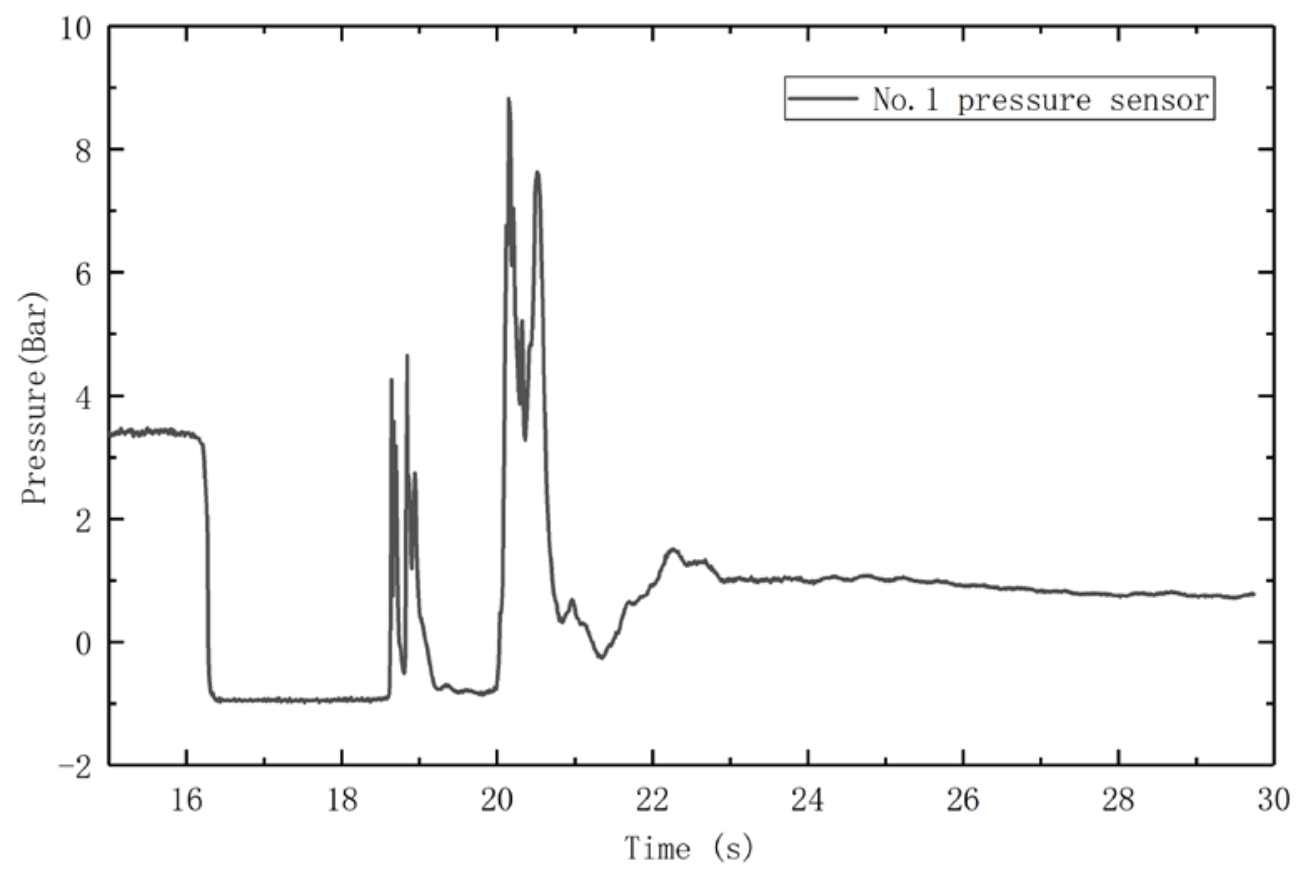

Figure 8. Instantaneous pressure of No. 1 pressure sensor.

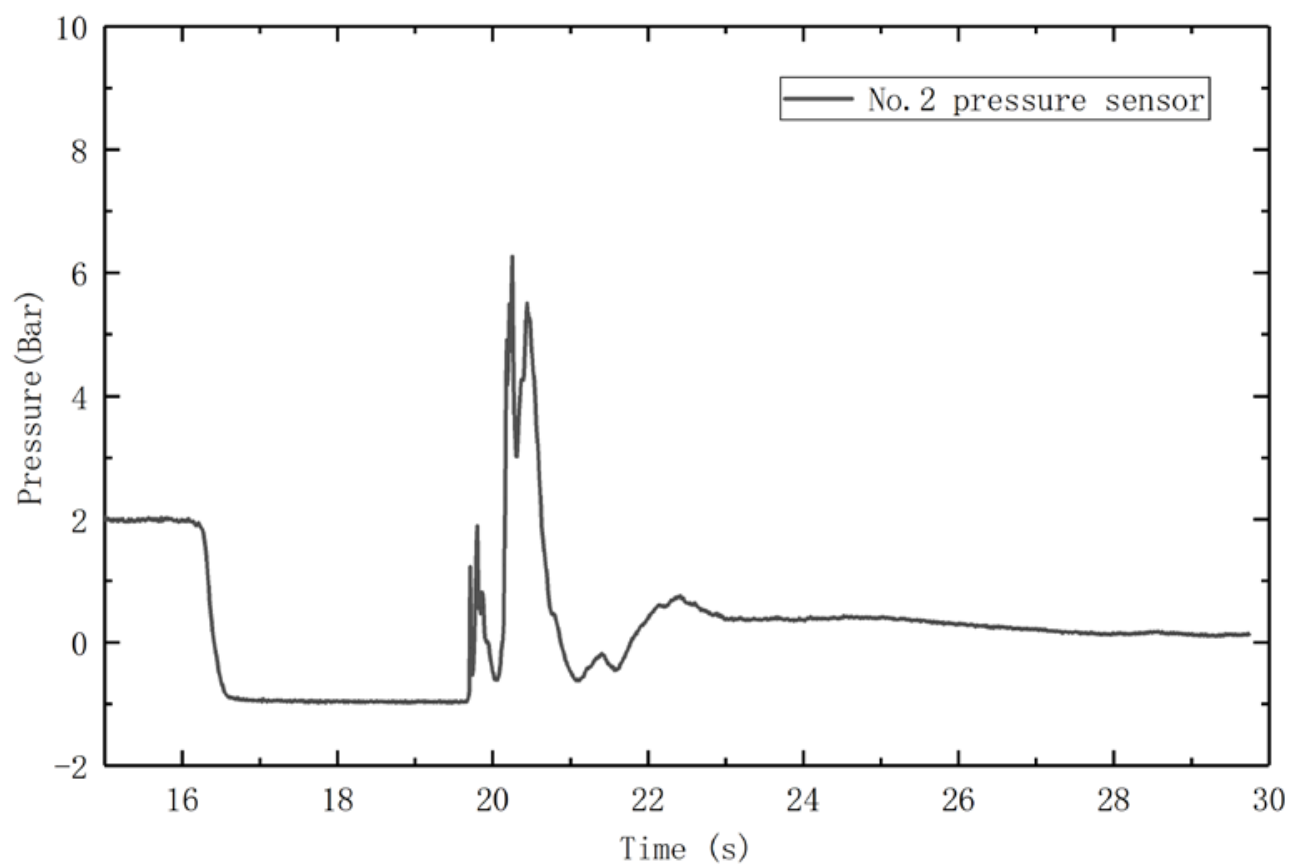

Figure 9. Instantaneous pressure of No. 2 pressure sensor.

\subsubsection{Effect of the Initial Flow Rate on the Water Hammer Pressure Increase and Cavity Length}

According to the experimental scheme, the opening of the manual valve is adjusted at the pump outlet to control the initial flow rate at $58,65,70.75,74$ and $77.7 \mathrm{~m}^{3} / \mathrm{h}$. The experimental phenomena and transient pressure due to the water hammer phenomenon were observed. The valve closing time was uniformly set to $1 \mathrm{~s}$. According to the experimental data, the influence of the initial flow rate on the water hammer pressure rise and maximum cavity length was analyzed.

For the same valve closing conditions (valve closing time was set to $1 \mathrm{~s}$ ), the water hammer pressure increase in the horizontal observation tube segment (point 1 ) at the outlet of the quick-closing valve and in the observation tube segment (point 2) at the knee of the tubeline was monitored. Based on 
the experimental data, when the initial flow rate increased, the water hammer increased the pressure at points 1 and 2. At an initial flow rate of $58 \mathrm{~m}^{3} / \mathrm{h}$, the maximum pressure at pressure measuring point 1 and measuring point 2 was 6.1 bar and 4.2 bar, respectively. At an initial flow rate of $70.75 \mathrm{~m}^{3} / \mathrm{h}$, the maximum pressure at pressure measuring point 1 and pressure measuring point 2 was 7.3 bar and 5.1 bar, respectively. At an initial flow rate of $77 \mathrm{~m}^{3} / \mathrm{h}$, the maximum pressure at pressure measuring point 1 and pressure measuring point 2 were 8.95 bar and 6.5 bar, respectively (Figure 10). Details are provided in Table $\mathrm{S} 7$ of the supplementary information.

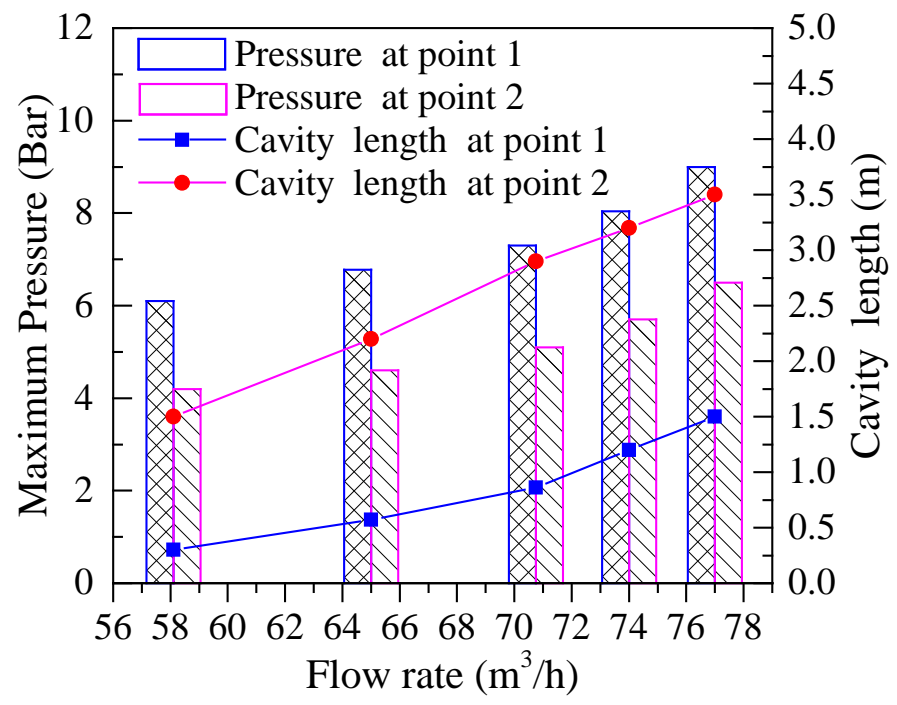

Figure 10. Relationship among the initial flow rate, measured maximum pressure, and cavity length.

In the experiments, a high-speed camera was adopted to record the length and shape of the flow interruption cavity. The length of the flow interruption cavity in the horizontal observation tube segment (point 1) at the outlet of the quick-closing valve and in the observation tube segment (point 2) at the knee of the tubeline was measured. According to the experimental data, when the initial flow rate increased, the length of the flow interruption cavity increased at points 1 and 2 . At an initial flow rate of $58 \mathrm{~m}^{3} / \mathrm{h}$, the cavity at point 1 and point 2 was $0.35 \mathrm{~m}$ and $1.5 \mathrm{~m}$, respectively. At an initial flow rate of $70.75 \mathrm{~m}^{3} / \mathrm{h}$, the cavity at point 1 and point 2 were $0.86 \mathrm{~m}$ and $2.9 \mathrm{~m}$, respectively. At an initial flow rate of $77 \mathrm{~m}^{3} / \mathrm{h}$, the cavity at point 1 and point 2 were $1.5 \mathrm{~m}$ and $3.5 \mathrm{~m}$, respectively (Figure 10).

When the initial flow rate is gradually increased, different hydraulic transition flow states may occur in the tubeline system. The basic reason for a pressure transient in pressurized tube flow is a sudden change in the flow velocity in the tube. According to the Joukowsky equation [14,15], the faster the flow rate changes, the greater the water hammer pressure. Therefore, when the valve closing time is the same, the greater the initial flow, the greater the water hammer pressure. In the experiments, due to the fast closing of the valve, the flow rate in the tube rapidly decreased, which caused a rapid drop in pressure. When the pressure was reduced to the liquid vaporization pressure, the flow state was determined by the remaining flow. If the residual flow is sufficient, complete flow interruption can occur. If the residual flow is low or even approaches 0 , incomplete flow interruption or atomized vaporization might occur. If the vaporization pressure is maintained for a short time, it is difficult to observe the atomized vaporization phenomenon. The residual flow depends on the initial flow rate. Therefore, the larger the initial flow rate, the longer the length of the cavity.

According to the above analysis, whether the interruption position occurred in the horizontal tube segment (point 1) or at the knee of the tubeline (point 2), with an increase in the initial flow rate, the cavity length and pressure increase both increased. 


\subsubsection{Effect of the Valve Closing Speed on the Water Hammer Pressure Increase and Cavity Length}

According to the experimental scheme, the opening of the manual valve at the pump outlet was altered to control the initial flow rate at $70 \mathrm{~m}^{3} / \mathrm{h}$. The valve closing times were set to $0.6,0.7,0.8,0.9$, 0.95 , and $1 \mathrm{~s}$. The water hammer-related pressurization of the horizontal observation tube segment (point 1) at the outlet of the quick-closing valve and that of the observation tube segment (point 2) at the knee of the tubeline were measured. Based on the experimental data, it was observed that when the valve closing speed increased, the pressure increase due to the water hammer increased at points 1 and 2 .

At a valve closing time of $0.6 \mathrm{~s}$, the maximum pressure at pressure measuring point 1 and pressure measuring point 2 was 8.83 bar and 6.17 bar, respectively. At a valve closing time of $0.9 \mathrm{~s}$, the maximum pressure at pressure measuring point 1 and pressure measuring point 2 was 7.69 bar and 5.37 bar, respectively. At a valve closing time of $1 \mathrm{~s}$, the maximum pressure at pressure measuring point 1 and pressure measuring point 2 were 7.21 bar and 5.08 bar, respectively. The shape of the water column at point 2 indicated that a short closing valve time corresponded to a steep collapse surface curve, and the higher the collapse speed was, the larger the pressure boost was (Figure 11).

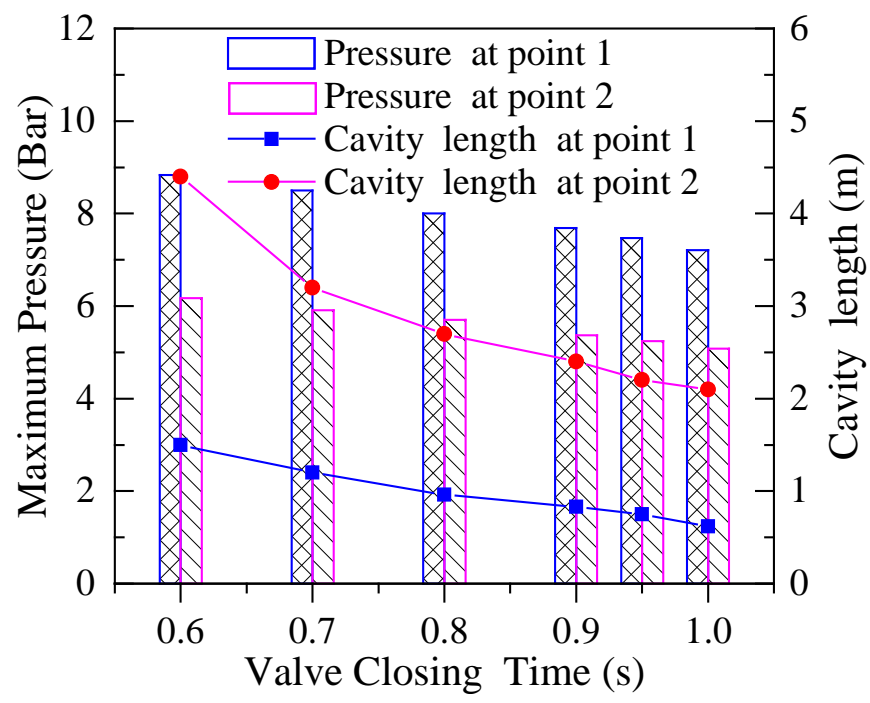

Figure 11. Relationship among the valve closing time, measured maximum pressure, and cavity length.

The cavity length measured in the experiments revealed that with the same initial flow conditions $\left(70 \mathrm{~m}^{3} / \mathrm{h}\right)$ at point 1 (No. 1 pressure measuring point) or point 2 (No. 2 pressure measuring point) and an increase in the valve closing time, the length of the flow interruption cavity decreased. At a valve closing time of $0.6 \mathrm{~s}$, the length of the cavity at point 1 and point 2 was $1.5 \mathrm{~m}$ and $4.4 \mathrm{~m}$, respectively. At a valve closing time of $0.9 \mathrm{~s}$, the length of the cavity at point 1 and point 2 was $0.83 \mathrm{~m}$ and $2.4 \mathrm{~m}$, respectively. At a valve closing time of $1 \mathrm{~s}$, the length of the cavity at point 1 and point 2 was $0.62 \mathrm{~m}$ and $2.1 \mathrm{~m}$, respectively (Figure 11). Details are provided in Table S7 of the supplementary information.

When the valve closing time is gradually increased, different hydraulic transition flow states may occur in the tubeline system. The transient value of pressure in the pressure pipe flow is related to the variation of the velocity in the pipe and the action time [1,5]. With the extension of valve closing time, the reflection wave superposition effect is obvious. Therefore, with the extension of valve closing time, the reflected wave reduces the forward water hammer wave, resulting in the decrease of water hammer pressure and cavity length.

According to the above analysis, whether the interruption position occurred in the horizontal tube segment or at the knee of the tubeline, with an increase in the valve closing speed, both the cavity length and the pressure increase. 


\section{Conclusions}

In the experiments, four water column separation (interruption) states were observed.

(1) The hydraulic transient process was affected by the initial flow rate, valve closing speed, and interruption position.

(2) The transient states observed in the horizontal tubeline (point 1) included the completely atomized vaporization state, the state with both flow interruption cavity and atomized vaporization zones, and the complete flow interruption state. The transient states observed at the knee of the tubeline (point 2) included complete and incomplete flow interruption states (or only a few bubbles were present).

(3) The flow state was determined by the remaining flow. If the residual flow rate was high enough, complete flow interruption would occur. If the residual flow rate was low or even approached 0 , incomplete flow interruption or atomized vaporization might occur. If the vaporization pressure was maintained for a short time, it was difficult to observe the atomized vaporization phenomenon. The residual flow velocity was affected by the gravity gradient, and the distribution of the residual flow velocity across the entire tubeline cross-section was not uniform. With a decrease in distance from the top of the tube, the residual flow velocity increased, and conversely, with an increase in distance from the top of the tube, the residual flow velocity decreased. Therefore, the flow interruption cavity exhibited a sloped surface.

(4) Whether the interruption position occurred in the horizontal tube segment or at the knee of the tubeline, with an increase in the initial flow velocity, the cavity length and pressure increase both increased. Moreover, with an increase in the valve closing speed, the cavity length and the pressure increase both increased.

Supplementary Materials: The following are available online at http://www.mdpi.com/2073-4441/12/9/2566/s1, Table S1. List of main materials and equipment, Table S2. Measurement of wave velocity of galvanized steel pipe, Table S3. Determination of wave velocity of plexiglass tube, Table S4. Resistance coefficient measurement of valve closing process, Table S5. Measurement of resistance coefficient during valve opening, Table S6. Valve resistance coefficient, Table S7. Water hammer pressure and cavity length under different initial flow rates and valve closing times, Figure S1. Pressure sensor, Figure S2. Electromagnetic flowmeter, Figure S3. Data acquisition card, Figure S4. Quick closing control valve, Figure S5. Speed regulating motor control box, Figure S6. High-speed camera, Figure S7. Fitting curve of valve resistance coefficient.

Author Contributions: Formal analysis, L.Z. (Li Zhao), Y.Y., and T.W.; Funding acquisition, Q.W. and L.Z. (Liang Zhou); Writing—original draft, L.Z. (Li Zhao), W.H., and R.W.; Data curation, T.W. and P.W. All authors have read and agreed to the published version of the manuscript.

Funding: This research was jointly supported by the Basic Research Program of Natural Science of Shaanxi Province (2019JQ-913) and the Research Fund Project of Xi'an Aeronautical University: "Research on the characteristics of opening flow coefficient of building rooms" (No.: 2017ky0210) and an experimental study on the performance improvement of a box-type bidirectional surge tank (No.: 2019 HX 045).

Conflicts of Interest: The authors declare no conflict of interest.

\section{References}

1. Bergant, A.; Simpson, A.R.; Tijsseling, A.S. Water hammer with column separation: A historical review. J. Fluids Struct. 2006, 22, 135-171. [CrossRef]

2. Ghidaoui, M.S. On the fundamental equations of water hammer. Urban Water J. 2004, 1, 71-83. [CrossRef]

3. Xiong, S.Y.; Guan, X.W.; Jin, Z. Various column separation and comprehensive protection of disconnection water hammer problems and design example. Water Supply Drain. J. 2003, 29, 1-5.

4. Ali, N.A.; Mohamed, H.I.; El-Darder, M.E.; Mohame, A.A. Analysis of transient flow phenomenon in pressurized pipes system and methods of protection. J. Eng. Sci. Assiut Univ. 2010, 38, 323-342.

5. Lema, M.; Peña, F.L.; Buchlin, J.-M.; Rambaud, P.; Steelant, J. Analysis of fluid hammer occurrence with phase change and column separation due to fast valve opening by means of flow visualization. Exp. Therm. Fluid Sci. 2016, 79, 143-153. [CrossRef] 
6. Luvizotto, E., Jr.; Koelle, E.; Andrade, J.G.P. Complete Operational Simulation of Pumped Storage Schemes; American Society of Civil Engineers: New York, NY, USA, 1995.

7. Wang, L.; Yue, X.; Chong, D.; Chen, W.; Yan, J. Experimental investigation on the phenomenon of steam condensation induced water hammer in a horizontal pipe. Exp. Therm. Fluid Sci. 2018, 91, 451-458. [CrossRef]

8. Riedelmeier, S.; Becker, S.; Schlücker, E. Measurements of junction coupling during water hammer in piping systems. J. Fluids Struct. 2014, 48, 156-168. [CrossRef]

9. Maryono, A.; Suhanan, S.; Kurniawan, A.; Alatas, M.; Akhita, M.R.; Wibisono, A.B. Experimental Study of Water Hammer Phenomena in Drinking Water Pipeline Distribution Using Video Camera Method. Int. J. Sci. Eng. Res. 2013, 4, 1-8.

10. Zhao, L.; Yang, Y.; Wang, T.; Zhou, L.; Li, Y.; Zhang, M. A Simulation Calculation Method of a Water Hammer with Multpoint Collapsing. Energies 2020, 13, 1103. [CrossRef]

11. Kim, S.-G.; Lee, K.-B.; Kim, K.-Y. Water hammer in the pump-rising pipeline system with an air chamber. J. Hydrodyn. 2014, 26, 960-964. [CrossRef]

12. Sonde, E.; Chaise, T.; Boisson, N.; Nélias, D. Modeling of cavitation peening: Jet, bubble growth and collapse, micro-jet and residual stresses. J. Mater. Process. Technol. 2018, 262, 479-491. [CrossRef]

13. Brunone, B.; Morelli, L. Automatic Control Valve-Induced Transients in Operative Pipe System. J. Hydraul. Eng. 1999, 125, 534-542. [CrossRef]

14. Zhao, L.; Yang, Y.S.; Lu, Y.D. Cavity water hammer characteristic test on two places. J. Adv. Sci. Technol. Water Resour. 2016, 36, 36-39.

15. Zhang, Y.; Miao, M.; Ma, J. Analytical study on water hammer pressure in pressurized conduits with a throttled surge chamber for slow closure. Water Sci. Eng. 2010, 3, 174-189.

(C) 2020 by the authors. Licensee MDPI, Basel, Switzerland. This article is an open access article distributed under the terms and conditions of the Creative Commons Attribution (CC BY) license (http://creativecommons.org/licenses/by/4.0/). 